\title{
The diagnostic utility of exome sequencing in Joubert syndrome and related disorders
}

Yoshinori Tsurusaki, Yasuko Kobayashi, Masataka Hisano, Shuichi Ito, Hiroshi Doi, Mitsuko Nakashima, Hirotomo Saitsu, Naomichi Matsumoto and Noriko Miyake

Journal of Human Genetics (2015) 60, 651; doi:10.1038/jhg.2015.86

Correction to: Journal of Human Genetics (2013) 58, 113-115; doi: 10.1038/jhg.2012.117; published online 4 October 2012

Since the publication of the above article, the authors have noticed an error in the description of mutation.
The mutation described as c.6012-12A $>\mathrm{T}$ in the main text and Figure 1a should have been c.6012-12T $>$ A.

This correction does not alter the results and their interpretation as discussed in the paper.

The authors would like to apologize for this mistake. 